International Journal of Pure and Applied Mathematics

Volume 106 No. 2 2016, 429-442

ISSN: 1311-8080 (printed version); ISSN: 1314-3395 (on-line version)

url: http://www.ijpam.eu

doi: 10.12732 /ijpam.v106i2.7

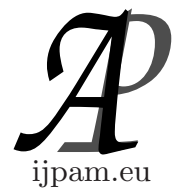

\title{
ON LOCAL SPECTRAL PROPERTIES OF $\lambda$-COMMUTING OPERATORS
}

\author{
Abdelaziz Tajmouati ${ }^{1}$, Abdeslam El Bakkali ${ }^{2}$, M.B. Mohamed Ahmed ${ }^{3}$ \\ ${ }^{1,3}$ Sidi Mohamed Ben Abdellah University \\ Faculty of Sciences Dhar El Marhaz \\ Fez, MOROCCO \\ ${ }^{2}$ Chouaib Dokkali University \\ Faculty of Sciences \\ El Jadida, MOROCCO
}

\begin{abstract}
Let $\mathcal{B}(X)$ be the Banach algebra of all bounded operators on a complex Banach space $X$, for a scalar $\lambda \in \mathbb{C}$ two operators $T, S \in \mathcal{B}(X)$ are said to $\lambda$-commute if $T S=\lambda S T$. If it holds, we show that $T S$ and $S T$ have many basic local spectral properties in common.
\end{abstract}

Key Words: spectrum, operator equation, $\lambda$-commutativity, local spectral properties

\section{Introduction}

Throughout we will denote by $\mathcal{B}(X)$ the Banach algebra of all linear operators on the complex Banach space $X$. For $T \in \mathcal{B}(X)$ we denote by $\sigma(T), N(T)$ and $R(T)$ the spectrum, the kernel and the range of $T$ respectively.

Recently many mathematicians have been attracted by the question: under what conditions if $T, S \in \mathcal{B}(X)$ there is $\lambda \in \mathbb{C}$ such that $T S=\lambda S T$ ?

Received: September 11, 2015

Published: February 10, 2016

$\S_{\text {Correspondence author }}$ (c) 2016 Academic Publications, Ltd.

url: www.acadpubl.eu 
It is well known that if $X$ is a Hilbert space and $\mathrm{T} \quad \lambda$-commutes with a compact operator, then $\mathrm{T}$ has a non-trivial hyperinvariant subspace [5].

In [2] Brooke, Busch and Pearson showed that for $T, S \in \mathcal{B}(X)$ satisfying $T S=\lambda S T$ then $\sigma(T S)=\sigma(S T)=\lambda \sigma(T S)$. If $T S$ is not quasinilpotent then necessary $|\lambda|=1$, and if $T$ or $S$ is self-adjoint then $\lambda \in \mathbb{R}$. At 2004, Yang and Du gave a simple proofs and generalizations of this results, particulary they proved that if $T S=\lambda S T$ then $T S$ is bounded below if and only if both $T$ and $S$ are bounded below [9, theorem 2.5]. Schmoeger in [8] generalized this results to hermitian or normal elements of a complex Banach algebra.

Cho, Duggal, Harte and ôta generalized some Schmoeger's results and they gave the new characterization of a commutativity of Banach space operators [3, theorem 2.4 and theorem 2.2].

In [4] where $X$ is a complex Hilbert space, Conway and Prajitura characterized the closure and the interior of the set of operators that $\lambda$-commute with a compact operator.

At 2011, Zhang, Ohwada and Cho have studied the properties of Hilbert space operators that $\lambda$-commute with a paranormal operator $[10$, theorem 1 and theorem 3].

In the present paper, our aim is to study some properties and concepts in local spectral theory for Banach space operators satisfying the $\lambda$-commutativity.

For $T \in \mathcal{B}(X)$, let the following notations, for detail see [1], [6], and [7]:

The spectrum of $T$

$$
\sigma(T)=\{\lambda \in \mathbb{C}: T-\lambda \text { is not invertible }\},
$$

The left spectrum

$$
\sigma_{l}(T)=\{\lambda \in \mathbb{C}: T-\lambda \text { is not left invertible }\},
$$

The right spectrum

$$
\sigma_{r}(T)=\{\lambda \in \mathbb{C}: T-\lambda \text { is not The right spectrum }\},
$$

The left or right spectrum

$$
\sigma_{l r}(T)=\{\lambda \in \mathbb{C}: T-\lambda \text { is not left or right inevertible }\},
$$

The ponctual spectrum

$$
\sigma_{p}(T)=\{\lambda \in \mathbb{C}: T-\lambda \text { is not injective }\},
$$

The surjective spectrum

$$
\sigma_{s u}(T)=\{\lambda \in \mathbb{C}: T-\lambda \text { is not surjective }\},
$$


The compression spectrum

$$
\sigma_{\text {com }}(T)=\{\lambda \in \mathbb{C}: R(T-\lambda) \text { is not dense in } X\}
$$

The approximate point spectrum

$$
\sigma_{a p}(T)=\left\{\lambda \in \mathbb{C} ; \exists\left(x_{n}\right)_{n \in \mathbb{N}} \text { of } X \text { such that }\left\|x_{n}\right\|=1 \text { and }(T-\lambda) x_{n} \rightarrow 0\right\} .
$$

Recall that $T$ has the single-valued extension property (SVEP) at $\lambda \in \mathbb{C}$ if for any neighborhood $U_{\lambda}$ of $\lambda$ the only analytical function of $f: U_{\lambda} \rightarrow X$ satisfying $(T-\mu) f(\mu)=x \forall \mu \in U_{\lambda}$ is the null function $f \equiv 0$.

We set

$$
\mathcal{S}(T)=\{\lambda \in \mathbb{C}: T \text { does not have SVEP at } \lambda\} .
$$

We say that $T$ has $\mathrm{SVEP}$ if $\mathcal{S}(T)=\emptyset$.

The local resolvent $\rho_{T}(x)$ of $T$ at $x \in X$ is defined as the set of all $\lambda \in \mathbb{C}$ such that there exists a neighborhood $U_{\lambda}$ of $\lambda$ and $f: U_{\lambda} \rightarrow X$ such that $(T-\mu) f(\mu)=x$ for all $\mu \in U_{\lambda}$.

The local spectrum $\sigma_{T}(x)$ of $T$ at $x$ is defined as $\sigma_{T}(x)=\mathbb{C} \backslash \rho_{T}(x)$.

Note that the local analytical solution of the equation given in the definition of the local resolvent will be unique if $T$ has SVEP [6].

For any subset $F$ of $\mathbb{C}$, The local spectral space of $T$ associated with $F$ is defined by

$$
X_{T}(F)=\left\{x \in X: \sigma_{T}(x) \subset F\right\} .
$$

Obviously $X_{T}(F)$ is a hyper-invariant space by $T$, but not necessarily closed.

Recall that $T$ has the property of Dunford $(\mathrm{C})$ if $X_{T}(F)$ is a closed set for every closed set $F$ of $\mathbb{C}$.

We denote by $\mathcal{O}(U, X)$ the Frchet algebra of all analytic functions from the open set $U$ to $X$ with the topology of uniform convergence on the compact subset in $U$.

We say that $T$ satisfies the Bishop's property $(\beta)$ at $\lambda \in \mathbb{C}$ if there exists $r>0$, for every open set $U \subset D(\lambda, r)$ and for any sequence $\left\{f_{n}\right\}_{n=1}^{\infty} \subset \mathcal{O}(U, X)$ such that $\lim _{n \rightarrow \infty}(T-\mu) f_{n}(\mu)=0$ in $\mathcal{O}(U, X)$, then $\lim _{n \rightarrow \infty} f_{n}(\mu)=0$ in $\mathcal{O}(U, X)$.

$$
\sigma_{\beta}(T)=\{\lambda \in \mathbb{C}: T \text { does not satisfy the property }(\beta)\}
$$

$T$ is said satisfy the property $(\beta)$ if $\sigma_{\beta}(T)=\emptyset$

We say that $T$ has the decomposition property $(\delta)$ if $T^{*}$ satisfies property $(\beta)$. 
$T$ is said decomposable on Foias's sense if and only if $T$ satisfies $(\beta)$ and $(\delta)$.

We have the following implications: Property $(\beta) \Rightarrow$ Dunford property $(\mathrm{C})$ $\Rightarrow$ SVEP.

For every closed set $F$ of $\mathbb{C}$, the global spectral subset $\mathcal{X}_{T}(F)$ is defined as the set of all point $x \in X$ such that there exists an analytic function $f: \mathbb{C} \backslash F \rightarrow$ $X$ satisfying $(T-\lambda) f(\lambda)=x$ for all $\lambda \in \mathbb{C} \backslash F$.

Clearly $\mathcal{X}_{T}(F)$ is a hyper invariant subspace of $T$ and $\mathcal{X}_{T}(F) \subset X_{T}(F)$. In addition we obtain the equality $\mathcal{X}_{T}(F)=X_{T}(F)$ for every closed set $F$ of $\mathbb{C}$ when $T$ has SVEP.

The algebraic core $C(T)$ of $T$ is the largest subspace $M$ of $X$ satisfying $T(M)=M$. In another way,

$$
C(T)=\left\{x \in X: \exists\left(x_{n}\right)_{n \geq 0} \subset X ; x_{0}=x, T x_{n}=x_{n-1} \forall n \in \mathbb{N}^{*}\right\} .
$$

and the analytical core $K(T)$ of $T$ is the set

$$
\begin{aligned}
& K(T)=\left\{x \in X: \exists\left(x_{n}\right)_{n \geq 0} \subset X, \text { and } \varepsilon>0 ; x_{0}=x,\right. \\
& \left.T x_{n}=x_{n-1},\left\|x_{n}\right\| \leq \varepsilon^{n}\|x\|, \forall n \in \mathbb{N}^{*}\right\} .
\end{aligned}
$$

$K(T)$ is the largest subspace of $X$ satisfying $T(M)=M$ and it can also be shown that

$$
K(T)=X_{T}(\mathbb{C} \backslash\{0\})=\left\{x \in X: 0 \in \rho_{T}(x)\right\} .
$$

Next, we need the following notations and concepts in Fredholm theory, see [1] and [7].

We denote by $N^{\infty}(T)=\bigcup_{n \in \mathbb{N}} N\left(T^{n}\right)$ the hyper-kernel of $T, R^{\infty}(T)=$ $\bigcap_{n \in \mathbb{N}} R\left(T^{n}\right)$ the hyper-range of $T$ and both the deficiency indices $\alpha(T)=$ $\operatorname{dim} N(T)$ and $\beta(T)=\operatorname{dim} R(T)$.

the ascent, the descent and the index of $T$ are respectively

$$
\begin{aligned}
\operatorname{asc}(T)= & \inf \left\{n \in \mathbb{N}: N\left(T^{n}\right)=N\left(T^{n+1}\right)\right\}, \\
\operatorname{des}(T)= & \inf \left\{n \in \mathbb{N}: R\left(T^{n}\right)=R\left(T^{n+1}\right)\right\}, \\
& \quad \operatorname{ind}(T)=\alpha(T)-\beta(T) .
\end{aligned}
$$

The ascent spectrum and the descent spectrum are the sets

$$
\begin{gathered}
\sigma_{\text {asc }}(T)=\{\lambda \in \mathbb{C}: \operatorname{asc}(T-\lambda)=\infty\}, \\
\sigma_{\text {des }}(T)=\{\lambda \in \mathbb{C}: \operatorname{des}(T-\lambda)=\infty\} .
\end{gathered}
$$


Let the sets of Fredholm operators, upper semi-Fredholm, lower semi-Fredholm, left semi-Fredholm, right semi-Fredholm, Weyl, upper semi-Weyl, lower semi-Weyl, left semi Weyl, right semi-Weyl, Browder, upper semi-Browder, lower semi-Browder, left semi-Browder and right semi-Browder respectively with their associated spectrums:

$$
\begin{gathered}
\Phi(X):=\{T \in \mathcal{B}(X): \alpha(T)<\infty \text { and } \beta(T)<\infty\}, \sigma_{e}(T), \\
\Phi_{+}(X):=\left\{T \in \mathcal{B}(X): \alpha(T)<\infty \text { and } R(T) \text { is closed, } \sigma_{S F^{+}}(T),\right. \\
\Phi_{-}(X):=\{T \in \mathcal{B}(X): \beta(T)<\infty\}, \sigma_{S F_{-}}(T), \\
\Phi_{l}(X):=\{T \in \mathcal{B}(X): \alpha(T)<\infty \text { and } R(T) \\
\text { is closed and admits an complemented in } X\}, \sigma_{l e}(T), \\
\Phi_{r}(X):=\{T \in \mathcal{B}(X): N(T) \\
\text { admits an complemented in } \mathrm{X} \text { and } \beta(T)<\infty\}, \sigma_{r e}(T), \\
\Phi_{0}(X):=\left\{T \in \Phi_{+}(X): \operatorname{ind}(T)=0\right\}, \sigma_{w}(T), \\
\Phi_{-}^{+}(X):=\left\{T \in \Phi_{-}(X): \operatorname{ind}(T) \geq 0\right\}, \sigma_{s w}(T), \\
\Phi_{l w}(X):=\left\{T \in \Phi_{l}(X): \operatorname{ind}(T) 0\right\}, \sigma_{l w}(T), \\
\Phi_{r w}(X):=\left\{T \in \Phi_{r}(X): \operatorname{ind}(T) \geq 0\right\}, \sigma_{r w}(T), \\
\Phi_{b}(X):=\{T \in \Phi(X): \text { asc }(T)=\operatorname{des}(T)<\infty\}, \sigma_{b}(T), \\
\Phi_{a b}(X):=\left\{T \in \Phi_{+}(X): \operatorname{asc}(T)<\infty\right\}, \sigma_{a b}(T), \\
\Phi_{s b}(X):=\left\{T \in \Phi_{-}(X): \operatorname{des}(T)<\infty\right\}, \sigma_{s b}(T), \\
\Phi_{l b}(X):=\left\{T \in \Phi_{l}(X): \operatorname{asc}(T)<\infty\right\}, \sigma_{l b}(T), \\
\Phi_{r b}(X):=\left\{T \in \Phi_{r}(X): \operatorname{des}(T)<\infty\right\}, \sigma_{r b}(T),
\end{gathered}
$$

Also we consider the following operators with their associated spectrum:

$$
\begin{aligned}
& R_{1}(X)=\left\{T \in \mathcal{B}(X): \operatorname{des}(T)<\infty, R\left(T^{\operatorname{des}(T)}\right) \text { is closed }\right\}, \sigma_{r D}(T), \\
& R_{2}(X)=\left\{T \in \mathcal{B}(X): \operatorname{asc}(T)<\infty, R\left(T^{\operatorname{des}(T)+1}\right) \text { is closed }\right\}, \sigma_{l D}(T), \\
& S F_{0}(T)=\left\{T \in \Phi_{+}(X) \cup \Phi_{-}(X): \alpha(T)=0 \text { or } \beta(T)=0\right\}, \sigma_{S F_{0}}(T),
\end{aligned}
$$




$$
D(X)=\left\{T \in \mathcal{B}(X): R(T) \text { is closed and } N(T) \subset R^{\infty}(T)\right\}, \sigma_{\text {se }}(T),
$$

Recall that $T \in \mathcal{B}(X))$ is Drazin reversible if there is $T^{D} \in \mathcal{B}(X)$ and some $k \in \mathbb{N}$

$$
T T^{D}=T^{D} T, T^{D} T T^{D}=T^{D}, T^{k+1} T^{D}=T^{k},
$$

The Drazin spectrum of $T$ is defined by

$$
\sigma_{D}(T)=\{\lambda \in \mathbb{C}: T-\lambda \text { is not Drazin reversible }\} .
$$

\section{Main Results}

We begin by the following theorem

Theorem 2.1. Let $T, S \in \mathcal{B}(X)$ and $\lambda \in \mathbb{C}^{*}$ such that

$$
T S=\lambda S T \text {. }
$$

Then we have :

1. $\sigma_{p}(T S)=\lambda \sigma_{p}(S T)$

2. $\sigma_{s u}(T S)=\lambda \sigma_{s u}(S T)$

3. $\sigma_{\text {com }}(T S)=\lambda \sigma_{\text {com }}(S T)$

4. $\sigma_{a p}(T S)=\lambda \sigma_{a p}(S T)$

5. $\sigma_{l}(T S)=\lambda \sigma_{l}(S T)$

6. $\sigma_{r}(T S)=\lambda \sigma_{r}(S T)$

Proof. 1. Let $\lambda \in \mathbb{C}^{*}$, it is clear that

$$
\begin{aligned}
\mu \in \sigma_{p}(T S) & \Leftrightarrow T S-\mu \text { is not injective } \\
& \Leftrightarrow \lambda S T-\mu=\lambda\left(S T-\frac{\mu}{\lambda}\right) \text { is not injective } \\
& \Leftrightarrow S T-\frac{\mu}{\lambda} \text { is not injective } \\
& \Leftrightarrow \frac{\mu}{\lambda} \in \sigma_{p}(S T)
\end{aligned}
$$

Hence $\sigma_{p}(T S)=\lambda \sigma_{p}(S T)$ 
2. Again if $\lambda \in \mathbb{C}^{*}$ then

$$
\begin{aligned}
\mu \notin \sigma_{s u}(T S) & \Leftrightarrow T S-\mu \text { is surjective } \\
& \Leftrightarrow R(T S-\mu)=X \\
& \Leftrightarrow R(\lambda S T-\mu)=R\left(\lambda\left(S T-\frac{\mu}{\lambda}\right)\right)=X \\
& \Leftrightarrow R\left(S T-\frac{\mu}{\lambda}\right)=X \\
& \Leftrightarrow \frac{\mu}{\lambda} \notin \sigma_{s u}(S T)
\end{aligned}
$$

Therefore $\sigma_{s u}(T S)=\lambda \sigma_{s u}(S T)$

3. Let $\lambda \in \mathbb{C}^{*}$ then

$$
\begin{aligned}
\mu \notin \sigma_{\text {com }}(T S) & \Leftrightarrow R(T S-\mu) \text { is dense in } X \\
& \Leftrightarrow \overline{R(T S-\mu)}=X \\
& \Leftrightarrow \overline{R(\lambda S T-\mu)}=\overline{R\left(\lambda\left(S T-\frac{\mu}{\lambda}\right)\right)}=X \\
& \Leftrightarrow \overline{R\left(S T-\frac{\mu}{\lambda}\right)}=X \\
& \Leftrightarrow \frac{\mu}{\lambda} \notin \sigma_{c o m}(S T)
\end{aligned}
$$

This shows that $\sigma_{c o m}(T S)=\lambda \sigma_{\text {com }}(S T)$

4. Let $\mu \in \sigma_{a p}(T S)$ then

$$
\begin{aligned}
& \mu \in \sigma_{a p}(T S) \Leftrightarrow \exists\left(x_{n}\right)_{n \in \mathbb{N}} \subset X \text { such that }\left\|x_{n}\right\|=1 \text { and } \\
& \lim _{n \rightarrow+\infty}(T S-\mu) x_{n}=0 \\
& \Leftrightarrow \exists\left(x_{n}\right)_{n \in \mathbb{N}} \subset X \text { such that }\left\|x_{n}\right\|=1 \text { and } \\
& \lim _{n \longrightarrow+\infty} \lambda\left(S T-\frac{\mu}{\lambda}\right) x_{n}=0 \\
& \Leftrightarrow \exists\left(x_{n}\right)_{n \in \mathbb{N}} \subset X \text { such that }\left\|x_{n}\right\|=1 \text { and } \\
& \lim _{n \rightarrow+\infty}\left(S T-\frac{\mu}{\lambda}\right) x_{n}=0 \\
& \Leftrightarrow \frac{\mu}{\lambda} \in \sigma_{a p}(S T) \text {. }
\end{aligned}
$$

Hence $\sigma_{a p}(T S)=\lambda \sigma_{a p}(S T)$

5. Let $\lambda \in \mathbb{C}^{*}$, then

$$
\mu \notin \sigma_{l}(T S) \Leftrightarrow T S-\mu \text { is left inversible }
$$




$$
\begin{aligned}
\Leftrightarrow & \exists T_{1} \in \mathcal{B}(X) \text { such that } \\
& T_{1}(T S-\mu)=I \\
\Leftrightarrow & \exists T_{1} \in \mathcal{B}(X) \text { such that } \\
& T_{1}(\lambda S T-\mu)=\left(\lambda T_{1}\right)\left(S T-\frac{\mu}{\lambda}\right)=I \\
\Leftrightarrow & \exists T_{1}^{\prime} \in \mathcal{B}(X) \text { such that } \\
& T_{1}^{\prime}\left(S T-\frac{\mu}{\lambda}\right)=I \text { with } T_{1}^{\prime}=\lambda T_{1} \\
\Leftrightarrow & S T-\frac{\mu}{\lambda} \text { is also left inversible } \\
\Leftrightarrow & \frac{\mu}{\lambda} \notin \sigma_{l}(S T)
\end{aligned}
$$

Hence $\sigma_{l}(T S)=\lambda \sigma_{l}(S T)$

6. Similarly.

We obtain the following corollary, see [2, lemma 2.1].

Corollary 2.1. Let $T, S \in \mathcal{B}(X)$ and $\lambda \in \mathbb{C}^{*}$ such that

$$
T S=\lambda S T \text {. }
$$

Then :

$$
\sigma(T S)=\lambda \sigma(S T) .
$$

Proof. Using theorem 2.1 we have

$$
\begin{aligned}
\sigma(T S) & =\sigma_{l}(T S) \cap \sigma_{r}(T S) \\
& =\left[\lambda \sigma_{l}(S T)\right] \cap\left[\lambda \sigma_{r}(S T)\right] \\
& =\lambda\left[\sigma_{l}(S T) \cap \sigma_{r}(S T)\right] \\
& =\lambda \sigma(S T) .
\end{aligned}
$$

We now establish the relationship between the local spectrum and $\beta$-spectrum for operators that $\lambda$-commute.

Theorem 2.2. Let $T, S \in \mathcal{B}(X), \mu \in \mathbb{C}$ and $\lambda \in \mathbb{C}^{*}$ such that

$$
T S=\lambda S T \text {. }
$$

Then we have: 
1. $\sigma_{T S}(x)=\lambda \sigma_{S T}(x)$

2. $\sigma_{\beta}(T S)=\lambda \sigma_{\beta}(S T)$

3. TS has SVEP at $\mu$ if and only if $S T$ has it at $\frac{\mu}{\lambda}$. Otherwise we have $\mathcal{S}(T S)=\lambda \mathcal{S}(S T)$

4. TS has SVEP if and only if $S T$ has it

Proof. 1. Suppose that $T S=\lambda S T$ and $\mu_{0} \notin \sigma_{T S}(x)$, then there exists a neighborhood $U$ of $\mu_{0}$ and $f \in \mathcal{O}(U, X)$ such that

$$
(T S-\mu) f(\mu)=x \text { for all } \mu \in U
$$

Since $T S=\lambda S T$, then

$$
\begin{aligned}
(T S-\mu) f(\mu)=x \text { for all } x \in U & \Leftrightarrow(\lambda S T-\mu) f(\mu)=x \text { for all } \mu \in U \\
& \Leftrightarrow \lambda\left(S T-\frac{\mu}{\lambda}\right) f(\mu)=x \text { for all } \mu \in U \\
& \Leftrightarrow\left(S T-\frac{\mu}{\lambda}\right)[\lambda f(\mu)]=x \text { for all } \mu \in U
\end{aligned}
$$

We define the following two bijections:

$S: X \rightarrow X$ with $S(x)=\lambda x$ and $s: \mathbb{C} \rightarrow \mathbb{C} \quad$ with $\quad s(z)=\lambda z$, then

$$
\lambda f(\mu)=(S \circ f)(\mu)=(S \circ f)\left(s\left(\frac{\mu}{\lambda}\right)\right) \text { for all } \mu \in U .
$$

Hence $\lambda f(\mu)=(S \circ f \circ s)\left(\frac{\mu}{\lambda}\right)$

And Since $\mu$ course the neighborhood $U$ of $\mu_{0}$ then $\frac{\mu}{\lambda}$ also course the neighborhood $V$ of $\frac{\mu_{0}}{\lambda}$, hence by replacing $\lambda f(\mu)$ by $(S \circ f \circ s)\left(\frac{\mu}{\lambda}\right)$ and by noting $g=S \circ f \circ s$ wich is analytic on $V$, we obtain:

$$
\begin{aligned}
(T S-\mu) f(\mu)=x \text { for all } x \in U & \Leftrightarrow \quad\left(S T-\frac{\mu}{\lambda}\right)[\lambda f(\mu)]=x \quad \text { for all } \mu \in U \\
& \left.\Leftrightarrow \quad\left(S T-\frac{\mu}{\lambda}\right)[S \circ f \circ s)\left(\frac{\mu}{\lambda}\right)\right]=x \\
& \quad \text { for all } \mu \in U \\
& \Leftrightarrow \quad\left(S T-\mu^{\prime}\right) g\left(\mu^{\prime}\right)=x \text { for all } \mu^{\prime} \in V
\end{aligned}
$$

Finally $\frac{\mu_{0}}{\lambda} \notin \sigma_{S T}(x)$ and therefore $\sigma_{T S}(x)=\lambda \sigma_{S T}(x)$

2. Let $\mu_{0} \in \sigma_{\beta}(T S)$, then there exists $r>0$ for every open set $U \subset D\left(\mu_{0}, r\right)$ and for all sequence $\left\{f_{n}\right\}_{n=1}^{\infty} \subset \mathcal{O}(U, X)$ such that

$$
\lim _{n \rightarrow \infty}(T S-\mu) f_{n}(\mu)=0 \Rightarrow \lim _{n \rightarrow \infty} f_{n}(\mu)=0 \text { in } \mathcal{O}(U, X)
$$


To show that $\frac{\mu_{0}}{\lambda} \in \sigma_{\beta}(S T)$, let $r^{\prime}>0, V \subset D\left(\frac{\mu_{0}}{\lambda}, r^{\prime}\right)$ and $\left(g_{n}\right)_{n \in \mathbb{N}}$ in $\mathcal{O}(U, X)$ such that $\lim _{n \rightarrow \infty}\left(S T-\mu^{\prime}\right) g_{n}\left(\mu^{\prime}\right)=0$. We have

$$
\begin{array}{ll} 
& \lim _{n \rightarrow \infty}\left(S T-\mu^{\prime}\right) g_{n}\left(\mu^{\prime}\right)=0 \text { in } \mathcal{O}(V, X) \\
\Leftrightarrow & \lim _{n \rightarrow \infty} \lambda\left(S T-\mu^{\prime}\right) g_{n}\left(\mu^{\prime}\right)=0 \text { in } \mathcal{O}(V, X) \\
\Leftrightarrow & \lim _{n \rightarrow \infty} \lambda\left(S T-\mu^{\prime}\right) g_{n}\left(\mu^{\prime}\right)=0 \text { in } \mathcal{O}(V, X) \\
\Leftrightarrow & \lim _{n \rightarrow \infty}\left(\lambda S T-\lambda \mu^{\prime}\right) g_{n}\left(\mu^{\prime}\right)=0 \text { in } \mathcal{O}(V, X) \\
\Leftrightarrow & \lim _{n \rightarrow \infty}\left(T S-\lambda \mu^{\prime}\right) g_{n}\left(\frac{1}{\lambda} \lambda \mu^{\prime}\right)=0 \text { in } \mathcal{O}(V, X) \\
\Leftrightarrow & \lim _{n \rightarrow \infty}\left(T S-\lambda \mu^{\prime}\right)\left[g_{n} \circ s^{-1}\right]\left(\lambda \mu^{\prime}\right)=0 \text { in } \mathcal{O}(V, X) \\
\Leftrightarrow & \lim _{n \rightarrow \infty} \lambda \mu^{\prime} \quad \lim _{n \rightarrow \infty}(T S-\mu)\left[g_{n} \circ s^{-1}\right](\mu)=0 \\
\Rightarrow & \lim _{n \rightarrow \infty} g_{n} \circ s^{-1}(\mu)=0 \text { in } \mathcal{O}(U, X) \\
\Rightarrow & \lim _{n \rightarrow \infty} g_{n}\left(\mu^{\prime}\right)=0 \text { in } \mathcal{O}(V, X)
\end{array}
$$

Hence $\frac{\mu_{0}}{\lambda} \in \sigma_{\beta}(S T)$. Similarly we can show the other inclusion.

Finally $\sigma_{\beta}(T S)=\lambda \sigma_{\beta}(S T)$

3. By the same argument as 2 .

4. As $\mathcal{S}(T S)=\lambda \mathcal{S}(S T)$ Then:

$T S$ has $S V E P \Leftrightarrow \mathcal{S}(T S)=\varnothing \Leftrightarrow \mathcal{S}(S T)=\varnothing$.

Lemma 2.1. Let $T, S \in \mathcal{B}(X)$ and $\lambda \in \mathbb{C}^{*}$ such that

$$
T S=\lambda S T \text {. }
$$

We have :

1. $R(T S)=R(S T)$, for all $\mu \in \mathbb{C} \quad R(T S-\mu)=R\left(S T-\frac{\mu}{\lambda}\right)$

2. $N(T S)=N(S T)$, for all $\mu \in \mathbb{C} \quad N(T S-\mu)=N\left(S T-\frac{\mu}{\lambda}\right)$

Proof. $\quad 1 . \Rightarrow)$ Let $y \in R(T S)$ then there exists $x \in X$ such that $T S(x)=$ $y$

$$
T S(x)=y \quad \Leftrightarrow \quad \lambda S T(x)=y
$$




$$
\begin{aligned}
& \Leftrightarrow S T(\lambda x)=y \\
& \Leftrightarrow \quad S T\left(x^{\prime}\right)=y \quad \text { with } \quad x^{\prime}=\lambda x
\end{aligned}
$$

Where $y \in R(S T)$.

$\Leftarrow)$ Similarly we show the reverse inclusion. Finally $R(T S)=R(S T)$

2. Let $x \in N(T S)$, then

$$
\begin{aligned}
x \in N(T S) & \Leftrightarrow T S(x)=0 \\
& \Leftrightarrow \lambda S T(x)=0 \\
& \Leftrightarrow S T(x)=0 \quad \forall \lambda \in \mathbb{C}^{*} \\
& \Leftrightarrow x \in N(S T)
\end{aligned}
$$

we then conclude that $N(T S)=N(S T)$.

As a straightforward consequence of Lemma 2.1 we easily obtain the following corollary

Corollary 2.2. Let $T, S \in \mathcal{B}(X)$ and $\lambda \in \mathbb{C}^{*}$ such that

$$
T S=\lambda S T
$$

Then we have for all $n \in \mathbb{N}^{*}$ :

1. $R\left((T S)^{n}\right)=R\left((S T)^{n}\right)$ and for all $\mu \in \mathbb{C} R\left[(T S-\mu)^{n}\right]=R\left[\left(S T-\frac{\mu}{\lambda}\right)^{n}\right]$

2. $N\left((T S)^{n}\right)=N\left((S T)^{n}\right)$ and for all $\mu \in \mathbb{C} N\left[(T S-\mu)^{n}\right]=N\left[\left(S T-\frac{\mu}{\lambda}\right)^{n}\right]$

3. $\alpha(T S)=\alpha(S T), \quad \beta(T S)=\beta(S T)$ and $\operatorname{ind}(T S)=\operatorname{ind}(S T)$

4. For all $\mu \in \mathbb{C} \alpha(T S-\mu)=\alpha\left(S T-\frac{\mu}{\lambda}\right), \quad \beta(T S-\mu)=\beta\left(S T-\frac{\mu}{\lambda}\right)$ and $\operatorname{ind}(T S-\mu)=\operatorname{ind}\left(S T-\frac{\mu}{\lambda}\right)$

5. $\operatorname{asc}(T S)=\operatorname{asc}(S T) \quad$ and $\operatorname{des}(T S)=\operatorname{des}(S T)$

6. For all $\mu \in \mathbb{C} \operatorname{asc}(T S-\mu)=\operatorname{asc}\left(S T-\frac{\mu}{\lambda}\right) \quad$ and $\quad \operatorname{des}(T S-\mu)=\operatorname{des}(S T-$ $\left.\frac{\mu}{\lambda}\right)$

7. $R^{\infty}(T S)=R^{\infty}(S T)$

8. For all $\mu \in \mathbb{C} R^{\infty}(T S-\mu)=R^{\infty}\left(S T-\frac{\mu}{\lambda}\right)$

9. $N^{\infty}(T S)=N^{\infty}(S T)$ 
10. For all $\mu \in \mathbb{C} N^{\infty}(T S-\mu)=N^{\infty}\left(S T-\frac{\mu}{\lambda}\right)$

We establish the following theorem, the proof is easily by using corollary 2.2

Theorem 2.3. Let $T, S \in \mathcal{B}(X)$ and $\lambda \in \mathbb{C}^{*}$ such that $T S=\lambda S T$. Then we have the following equalities :

1. $\sigma_{a s c}(T S)=\lambda \sigma_{a s c}(S T), \quad \sigma_{d e s}(T S)=\lambda \sigma_{d e s}(S T)$

2. $\sigma_{e}(T S)=\lambda \sigma_{e}(S T)$

3. $\sigma_{S F^{+}}(T S)=\lambda \sigma_{S F^{+}}(S T), \quad \sigma_{S F^{-}}(T S)=\lambda \sigma_{S F^{-}}(S T)$

4. $\sigma_{l e}(T S)=\lambda \sigma_{l e}(S T), \quad \sigma_{r e}(T S)=\lambda \sigma_{r e}(S T)$

5. $\sigma_{w}(T S)=\lambda \sigma_{w}(S T)$

6. $\sigma_{a w}(T S)=\lambda \sigma_{a w}(S T), \quad \sigma_{s w}(T S)=\lambda \sigma_{s w}(S T)$

7. $\sigma_{l w}(T S)=\lambda \sigma_{l w}(S T), \quad \sigma_{r w}(T S)=\lambda \sigma_{r w}(S T)$

8. $\sigma_{b}(T S)=\lambda \sigma_{b}(S T)$

9. $\sigma_{a b}(T S)=\lambda \sigma_{a b}(S T), \quad \sigma_{s b}(T S)=\lambda \sigma_{s b}(S T)$

10. $\sigma_{l b}(T S)=\lambda \sigma_{l b}(S T), \quad \sigma_{r b}(T S)=\lambda \sigma_{r b}(S T)$

11. $\sigma_{s e}(T S)=\lambda \sigma_{s e}(S T)$

12. $\sigma_{B F}(T S)=\lambda \sigma_{B F}(S T), \quad \sigma_{B W}(T S)=\lambda \sigma_{B W}(S T)$

13. $\sigma_{r D}(T S)=\lambda \sigma_{r D}(S T), \quad \sigma_{l D}(T S)=\lambda \sigma_{l D}(S T)$

14. $\sigma_{S F_{0}}(T S)=\lambda \sigma_{S F_{0}}(S T)$

The connection between the Drazin spectrum for operators satisfying the $\lambda$-commutativity is the following

Theorem 2.4. Let $T, S \in \mathcal{B}(X)$ and $\lambda \in \mathbb{C}^{*}$ such that $T S=\lambda S T$. Then

$$
\sigma_{D}(T S)=\lambda \sigma_{D}(S T)
$$


Proof. Let $\left.\mu \notin \sigma_{D}(T S){ }^{*}\right)$, as $T S=\lambda S T$ then we have the following equivalents :

$$
\begin{aligned}
& (*) \Leftrightarrow T S-\mu \text { is Drazin invertible } \\
& \Leftrightarrow \quad \exists R \in \mathcal{B}(X),(T S-\mu) R=R(T S-\mu), R(T S-\mu) R=R \\
& \text { and }(T S-\mu)^{n+1} R=(T S-\mu)^{n} \\
& \Leftrightarrow \quad(\lambda S T-\mu) R=R(\lambda S T-\mu), R(\lambda S T-\mu) R=R \\
& \text { and }(\lambda S T-\mu)^{n+1} R=(\lambda S T-\mu)^{n} \\
& \Leftrightarrow \quad\left(S T-\frac{\mu}{\lambda}\right)[\lambda R]=[\lambda R]\left(S T-\frac{\mu}{\lambda}\right),[\lambda R]\left(S T-\frac{\mu}{\lambda}\right) R=R, \\
& \lambda^{n+1}\left(S T-\frac{\mu}{\lambda}\right)^{n+1} R=\lambda^{n}\left(S T-\frac{\mu}{\lambda}\right)^{n} \\
& \Leftrightarrow \quad\left(S T-\frac{\mu}{\lambda}\right)[\lambda R]=[\lambda R]\left(S T-\frac{\mu}{\lambda}\right),[\lambda R]\left(S T-\frac{\mu}{\lambda}\right)[\lambda R]=[\lambda R], \\
& \left(S T-\frac{\mu}{\lambda}\right)^{n+1}[\lambda R]=\left(S T-\frac{\mu}{\lambda}\right)^{n} \\
& \Leftrightarrow S T-\frac{\mu}{\lambda} \text { is Drazin invertible } \\
& \Leftrightarrow \frac{\mu}{\lambda} \notin \sigma_{D}(S T) .
\end{aligned}
$$

Using the previous results we obtain the following properties on local spectral space, global spectral, analytical core and the property $(C)$ for operators satisfying the $\lambda$-commutativity.

Theorem 2.5. Let $T, S \in \mathcal{B}(X)$ and $\lambda \in \mathbb{C}^{*}$ such that $T S=\lambda S T$. Then

1. $X_{T S}(F)=X_{S T}\left(\frac{F}{\lambda}\right)$ and $\mathcal{X}_{T}(F)=\mathcal{X}_{T}\left(\frac{F}{\lambda}\right)$

2. $T S$ has the property $(C)$ if and only if $S T$ also has the property $(C)$

3. $K(T S)=K(S T)$

\section{References}

[1] P.Aiena. Fredholm and Local Spectral Theory with Applications to Multipliers. Kluwer.Acad.Press,2004.

[2] J.A. Brooke, P. Busch, D.B. Pearson. Commutativity up to a factor of bounded operators in complex Hilbert space. Proc. R. Soc. Lond. A 458 (2002), 109-118.

[3] M. Сho, B. Duggal, R.E. Harte, S. Ôta. Operator Equation $A B=\lambda B A$ International Math. Forum, 5(2010), 2629-2637. 
[4] J.B. Conway, Gabriel Prajitura . On $\lambda$-commuting operator. Studia Mathematica 166 (1) (2005).

[5] V. LAURIC. Operators $\alpha$-commuting with a compact operator. Proc. Amer. Math. Soc. 125(1997), 2379-2384.

[6] K.B.Laursen, M.M.Neumann.An introduction to Local Spectral Theoryin: London Mathematical Society Monograph, New series, Vol. 20, Clarendon Press, Oxford, 2000

[7] V. MüLler. Spectral Theory of Linear Operators and Spectral Systems in Banach Algebras 2nd edition. Oper. Theory Advances and Applications. vol 139 (2007).

[8] C. Schmoeger. Commutativity up to a factor in Banach algebras. Demonst. Math. 38(2005) 895-900

[9] J. YANG, H.-K. Du. A note on commutativity up to a factor of bounded operators. Proc. Amer. Math. Soc. 132(2004), 1713-1720.

[10] L. Zhang, T. Ohwada, M. Cho. On $\lambda$-Commuting Operators. International Mathematical Forum, Vol. 6, 2011, no. 34, 1685 - 1690. 